\title{
Magnetohydrodynamic micropolar fluid flow in presence of nanoparticles through porous plate: A numerical study
}

\author{
S.M. Arifuzzaman ${ }^{1}$, Md. Farid Uddin Mehedi ${ }^{2}$, Abdullah Al-Mamun ${ }^{3}$, Pronab Biswas ${ }^{1}$, Md. Rafiqul Islam ${ }^{4}$, Md. Shakhaoath \\ $\mathrm{Khan}^{5^{*}}$ \\ ${ }^{1}$ Mathematics Discipline, Khulna University, Khulna 9208, Bangladesh \\ ${ }^{2}$ Department of Thermal Science \& Energy Engineering, University of Science \& Technology of China, Anhui 230026, China \\ ${ }^{3}$ Physics Discipline, Khulna University, Khulna-9208, Bangladesh \\ ${ }^{4}$ Department of Mathematics, Pabna University of Science \& Technology, Pabna 6600, Bangladesh \\ ${ }^{5}$ School of Engineering, RMIT University, GPO Box 2476, Melbourne 3001, VIC, Australia
}

Corresponding Author Email: mohammad.shakhaoath.khan@rmit.edu.au

https://doi.org/10.18280/ijht.360321

Received: 11 December 2017

Accepted: 15 September 2018

\section{Keywords:}

micropolar fluid, nanoparticles, radiation absorption, chemical reaction, thermal radiation, stability and convergence analysis

\begin{abstract}
This study numerically investigates Magnetohydrodynamic (MHD) convective and chemically reactive unsteady micropolar fluid flow with nanoparticles through the vertical porous plate with mass diffusion, thermal radiation, radiation absorption and heat source. A flow model is established by employing the well-known boundary layer approximations. To obtain the nonsimilar equation, the boundary layer governing equations including continuity, momentum, energy and concentration balance were nondimensionalised by usual transformation. A nonsimilar approach is applied to the flow model. To optimize the parametric values, the stability and convergence analysis (SCA) have been analysed for the Prandtl number (Pr) and Lewis number (Le). It is observed that with initial boundary conditions, $U=V=T=C=0$ and for $\Delta \tau$ $=0.005, \Delta X=0.20$ and $\Delta Y=0.25$, the system converged at Prandtl number, $P_{r} \geq 0.356$ and Lewis number, $\mathrm{Le}_{\mathrm{e}} \geq 0.16$. The coupled non-linear partial differential equations are solved by explicit finite difference method (EFDM) and the numerical results have been calculated by Compaq Visual FORTRAN 6.6a. Evaluation of the thermal and momentum boundary layer thickness with isotherms and streamlines analysis of boundary layer flows have been shown for the thermal radiation parameter $(\mathrm{R})$. The effects of various parameters entering the problem on velocity, angular velocity, temperature and concentration are shown graphically.
\end{abstract}

\section{INTRODUCTION}

Micropolar fluids are well known as the fluids with microstructure. The theory of micropolar fluids introduced by Eringen [1] is one of the best theories of fluids to describe the structured fluids and these fluids consist of rigid particles which can rotate with their own spins and microrotations. The concentration laws of mass, momentum and fundamental relationships distinguish the fluid motion of the micropolar fluid and it's describing the effect of couple stress, spin-inertia and micromotion which are very important in micropolar fluids. After the investigation of micropolar theory, there are many researchers especially focus on industrial applications and extend the study in many ways to include various physical effects.

The summary of the theory of micropolar fluid lies in particle suspension [2], liquid crystals [3]; animal blood [4], exotic lubricants [5], etc. Recently, lots of researcher focus on physical and engineering problems which involves of micropolar fluid and mainly they concern on suction or injection in boundary conditions, heat transfer by free or mixed convection, stagnation point flow, the stretching / shrinking sheet problems, magnetohydrodynamics, velocity slip and even also the flow and heat transfer of micropolar fluid through a horizontal or vertical channel. Micropolar fluid plays a practical role in the biological science and in manufacturing, chemical and food industry, bio-medical science etc. An excellent review of the different applications of micropolar fluid mechanics was presented by Ariman et al. [6].

The importance of Boundary layer flow over a stretching in various engineering processes, as an example, materials manufactured by extrusion. a stretching sheet interacts with the ambient fluid both thermally and mechanically during this process. Crane [7] was initiated the study of boundary layer flow caused by a stretching surface. By a porous stretching sheet, the effect of surface conditions on the micropolar flow was studied by Kelson et al. [8]. Mohammadein et al. [9] examined the flow of micropolar fluid over a stretching sheet with prescribed wall heat flux, viscous dissipation and internal heat generation. Hussain et al. [10] presented a model-based analysis of micropolar nanofluid flow over a stretching surface. Nazar et al. [11-12] investigated the stagnation point and unsteady boundary layer flow over a stretching sheet in a micropolar fluid. Bhargava et al. [13] examined the flow of a mixed convection micropolar fluid driven by a porous stretching sheet with uniform section and later, Bhargava et al. [14] investigated the same flow of a micropolar flow over a nonlinearly stretching sheet. In many engineering activities, the process of suction is used such as thermal oil recovery, removal of reactants etc. Erickson et al. [15] and Fox et al. [16] was studied the effect of suction or injection at a stretching 
surface. In recent time, several works on the dynamic of the boundary layer flow over a stretching surface have appeared in the literature [17-19]. Unsteady forced bioconvection slip flow of a micropolar nanofluid from a stretching/shrinking sheet was studied by Latiff [20]. In the industrial process, the heat generation and absorption are an enormous phenomenon. Recently, Abel et al. [21] numerical analysed the hydromagnetic micropolar fluid flow due to horizontal/vertical stretching sheet using a shooting method. They highlighted a scientific approach for the choice of the missing initial values on which the convergence of the shooting method highly depends. Afterwards, Abbas et al. [22] analysed the heat transfer for stretching flow over a curved surface with a magnetic field. Naveed et al. [23] investigated hydromagnetic flow over an unsteady but it was curved stretching surface. Later, Naveed et al. [24] studied the magnetohydrodynamic flow of a micropolar fluid in the presence of thermal radiation over a curved stretching sheet. Boundary layer flow of magneto-micropolar nanofluid flow with Hall and ion-slip effects using variable thermal diffusivity was examined by Bilal [25].

In recent time, Arifuzzaman et al. [26] analyzed unsteady chemically reactive micropolar fluid through an infinite vertical plate with the influence of thermal radiation, porous medium, thermal and mass diffusion with heat and mass transfer and showed the velocity, angular velocity, temperature and concentration across the boundary layer. Afterwards, in presence of nanoparticle, Arifuzzaman et al. [27] studied chemically reactive viscoelastic fluid flow through the porous stretching sheet. Khan et al. [28], Biswas et al. [29] and Arifuzzaman et al. [30] investigated impacts of magnetic field and radiation absorption on mixed convective and radiative of Williamson fluid, Jeffery nanofluid and Maxwell fluid flow over a linear or vertical stretching sheet with stability and convergence analysis (SCA). Arifuzzaman et al. [31] investigated the momentum boundary layer and thermal boundary layer thickness with streamlines and isotherms variation of transient MHD natural convective and chemically reactive high-speed fluid flow through an oscillatory vertical porous plate in presence of heat and radiation absorption. The heat exchange in conditions of free convection for the heat radiator, Wernik et al. [33] validated the results of numerical simulations using thermovision for three heat flux values. Oravec et al. [34] have investigated the improvement of control performance and increase of energy savings using the soft-constrained robust MPC with integral action for a laboratory plate heat exchanger.

In this paper, our prime objective is to investigate naturally convective and chemically reactive unsteady micropolar fluid flow with nanoparticles through a vertical porous plate with mass diffusion, MHD, thermal radiation, radiation absorption and heat source. By using the well-known boundary layer approximations, a flow model is established.

- The governing systems of partial equations have been transformed to set of coupled ordinary differential equations with the help of suitable non-similar transformations.

- Coupled non-linear dimensionless flow equations have been solved numerically by EFDM.

- The accuracy of our method to study, the stability and convergence analysis for the Prandtl number (Pr) and Lewis number (Le) have been analyzed to determine the parametric values.

- The results have been discussed in detail to study and shown graphically with the influence of various non-dimensional governing parameters on velocity, temperature and concentration.

- Evaluation of the thermal and momentum boundary layer thickness with isotherms and streamlines analysis of boundary layer flows have been shown for the thermal radiation parameter $(\mathrm{R})$.

\section{MATHEMATICAL FLOW MODEL}

The fluid with both micro-rotation and micro-inertia properties is known as micropolar fluid. Unsteady heat and mass transfer flow of viscoelastic fluid along a semi-infinite vertical porous plate are considered in the presence of a uniform thermal radiation and magnetic field. The flow is in the $x$-direction which is taken along the plate in the upward $x$ direction and $y$-axis is normal to it. When the plate velocity $U(t)$ is given as $u=0$. In an initial step, it is considered that the plate, as well as the fluid particle, is at rest at the same temperature $T\left(=T_{\infty}\right)$ and the same concentration level $\mathrm{C}(=$ $\left.C_{\infty}\right)$ at all points. It is also assumed that a magnetic field $B_{y}=$ $B_{0}$ of uniform strength is applied normal to the flow region along the y-axis. When $S=0$, then $\bar{N}=0$ which represents no-spin condition i.e., the microelements in a concentrated particle flow close to the wall are not able to rotate. The case $S=1 / 2$ represents vanishing of the anti-symmetric part of the stress tensor and represents weak concentration. In a fine particle suspension of the particle, spin is equal to the fluid velocity at the wall. The case $S=1$ represents turbulent boundary layer flow.

Continuity Equation:

$\frac{\partial u}{\partial x}+\frac{\partial v}{\partial y}=0$

Momentum Equation,

$\frac{\partial u}{\partial t}+u \frac{\partial u}{\partial x}+v \frac{\partial u}{\partial y}=g \beta\left(T-T_{\infty}\right)+g \beta^{*}\left(C-C_{\infty}\right)$

$+\left(v+\frac{\chi}{\rho}\right) \frac{\partial^{2} u}{\partial y^{2}}+\frac{\chi}{\rho} \frac{\partial N}{\partial y}-\frac{\sigma^{\prime} B_{0}^{2} u}{\rho}-\frac{v}{K^{\prime}} u-\frac{b}{K^{\prime}} u^{2}$

Angular Momentum Equation,

$\frac{\partial \bar{N}}{\partial t}+u \frac{\partial \bar{N}}{\partial x}+v \frac{\partial \bar{N}}{\partial y}=\frac{\gamma}{\rho j}\left(\frac{\partial^{2} \bar{N}}{\partial y^{2}}\right)-\frac{\chi}{\rho j}\left(2 \bar{N}+\frac{\partial u}{\partial y}\right)$
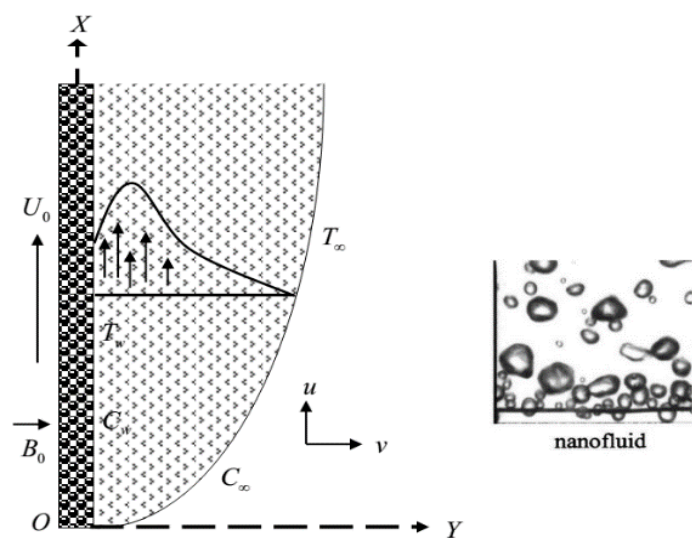

Figure A1. Physical configuration and coordinate system 
Energy Equation,

$$
\begin{aligned}
& \frac{\partial T}{\partial t}+u \frac{\partial T}{\partial x}+v \frac{\partial T}{\partial y}=\frac{\kappa}{\rho c_{p}} \frac{\partial^{2} T}{\partial y^{2}}+\frac{1}{c_{p}}\left(v+\frac{\chi}{\rho}\right) \\
& {\left[\left(\frac{\partial u}{\partial y}\right)^{2}+\left(\frac{\partial \bar{N}}{\partial y}\right)^{2}\right]+\frac{Q_{0}}{\rho c_{p}}\left(T-T_{\infty}\right)} \\
& +\frac{Q_{1}^{*}}{\rho c_{p}}\left(C-C_{\infty}\right)-\frac{1}{\rho c_{p}} \frac{\partial q_{r}}{\partial y}+\frac{D_{m} \kappa_{T}}{c_{s} c_{p}} \frac{\partial^{2} C}{\partial y^{2}} \\
& +\tau\left\{D_{B}\left(\frac{\partial T}{\partial y} \frac{\partial C}{\partial y}\right)+\frac{D_{T}}{T_{\infty}}\left(\frac{\partial T}{\partial y}\right)^{2}\right\}
\end{aligned}
$$

Concentration Equation,

$$
\frac{\partial C}{\partial t}+u \frac{\partial C}{\partial x}+v \frac{\partial C}{\partial y}=D_{B}\left(\frac{\partial^{2} C}{\partial y^{2}}\right)+\frac{D_{T}}{T_{\infty}} \frac{\partial^{2} T}{\partial y^{2}}-K_{c}\left(C-C_{\infty}\right)^{P}
$$

With boundary condition,

$$
\begin{aligned}
& u=0, \bar{N}=-S \frac{\partial u}{\partial y}, T=T_{w}, C=C_{w} \text { at } y=0 \\
& u=0, \bar{N} \rightarrow N_{\infty}, T \rightarrow T_{\infty}, C \rightarrow C_{\infty} \text { at } y \rightarrow \infty
\end{aligned}
$$

where, $u$ and $v$ denotes the velocity component, $B_{0}$ is the magnetic field component, $\beta$ is thermal expansion coefficient, $\beta^{*}$ is concentration expansion coefficient, $\sigma^{\prime}$ electric conductivity $T_{w}$ is the wall temperature, $C_{w}$ is the species concentration at the wall, $v$ is the kinematic viscosity, $\rho$ is density, $\kappa$ is thermal conductivity, $c_{p}$ is specific heat at constant pressure, $Q_{0}$ denotes the heat source, $Q_{1}^{*}$ denotes the radiation absorption, $q_{r}$ denotes the unidirectional radiative heat flux, $K_{C}$ is the chemical reaction, $D_{B}$ denotes Brownian diffusion coefficient, $D_{T}$ thermophoresis diffusion coefficient. The radiative heat flux term by using the Rosseland approximation is given by, $q_{r}=-\frac{4 \sigma_{s}}{3 k_{e}} \frac{\partial T^{4}}{\partial y}$.

where, $\sigma_{s}$ denotes Stefan-Boltzmann constant and $k_{e}$ mean absorption coefficient, respectively. If temperature differences within the flow are sufficiently small and after neglecting higher order terms takes the form by, then the $q_{r}$ can be linearized by expanding $T^{4}$ into the Taylor series about $T_{\infty}$ .Then the equation (3) becomes the following form,

$$
\begin{aligned}
& \frac{\partial T}{\partial t}+u \frac{\partial T}{\partial x}+v \frac{\partial T}{\partial y}=\frac{\kappa}{\rho c_{p}} \frac{\partial^{2} T}{\partial y^{2}}+\frac{v}{c_{p}}\left[\left(\frac{\partial u}{\partial y}\right)^{2}+\left(\frac{\partial \bar{N}}{\partial y}\right)^{2}\right] \\
& +\frac{Q_{0}}{\rho c_{p}}\left(T-T_{\infty}\right)+\frac{Q_{1}^{*}}{\rho c_{p}}\left(C-C_{\infty}\right)+\frac{16 \sigma_{s} T_{\infty}^{3}}{3 k_{e} \rho c_{p}} \frac{\partial^{2} T}{\partial y^{2}} \\
& +\frac{D_{m} \kappa_{T}}{c_{s} c_{p}} \frac{\partial^{2} C}{\partial y^{2}}+\tau\left\{D_{B}\left(\frac{\partial T}{\partial y} \frac{\partial C}{\partial y}\right)+\frac{D_{T}}{T_{\infty}}\left(\frac{\partial T}{\partial y}\right)^{2}\right\}
\end{aligned}
$$

From the governing equations (1) - (5) under the initial conditions and the boundary conditions are transformed into dimensionless form and the dimensionless quantities are,

$$
\begin{aligned}
& X=\frac{x U_{0}}{v}, Y=\frac{y U_{0}}{v}, U=\frac{u}{U_{0}}, V=\frac{v}{U_{0}}, \\
& \tau=\frac{t U_{0}^{2}}{v}, N=\frac{v \bar{N}}{U_{0}^{2}}, \theta=\frac{T-T_{\infty}}{T_{w}-T_{\infty}}, \phi=\frac{C-C_{\infty}}{C_{w}-C_{\infty}}
\end{aligned}
$$

So,

$x=\frac{X v}{U_{0}}, y=\frac{Y v}{U_{0}}, u=U U_{0}, t=\frac{\tau v}{U_{0}^{2}}, T=T_{\infty}+\theta\left(T_{w}-T_{\infty}\right) \quad$ and

$C=C_{\infty}+\phi\left(C_{w}-C_{\infty}\right)$

The dimensionless equations are obtained as follows:

Dimensionless Continuity Equation,

$\frac{\partial U}{\partial X}+\frac{\partial V}{\partial Y}=0$

Dimensionless Momentum Equation,

$\frac{\partial U}{\partial \tau}+U \frac{\partial U}{\partial X}+V \frac{\partial U}{\partial Y}==G_{r} \theta+G_{c} \phi+(1+\Gamma) \frac{\partial^{2} U}{\partial Y^{2}}$

$+\Gamma \frac{\partial \bar{N}}{\partial Y}-M U-\frac{1}{D_{a}} U-\frac{F_{s}}{D_{a}} U^{2}$

Dimensionless Angular Momentum Equation,

$\frac{\partial N}{\partial \tau}+U \frac{\partial N}{\partial X}+V \frac{\partial N}{\partial Y}=\Lambda \frac{\partial^{2} N}{\partial Y^{2}}-\lambda\left(2 N+\frac{\partial U}{\partial Y}\right)$

Dimensionless Energy Equation,

$\frac{\partial \theta}{\partial \tau}+U \frac{\partial \theta}{\partial Y}+V \frac{\partial \theta}{\partial Y}=\frac{1}{P_{r}}\left(1+\frac{16 R}{3}\right) \frac{\partial^{2} \theta}{\partial Y^{2}}$

$+Q \theta+Q_{1} \phi+E_{c}\left[\left(\frac{\partial U}{\partial Y}\right)^{2}+\left(\frac{\partial N}{\partial Y}\right)^{2}\right]+D_{u} \frac{\partial^{2} \phi}{\partial Y^{2}}$

$+N_{b}\left(\frac{\partial \theta}{\partial Y} \frac{\partial C}{\partial Y}\right)+N_{t}\left(\frac{\partial \theta}{\partial Y}\right)^{2}$

Dimensionless Concentration Equation,

$\frac{\partial \phi}{\partial \tau}+U \frac{\partial \phi}{\partial X}+V \frac{\partial \phi}{\partial Y}=\frac{1}{L_{e}}\left[\frac{\partial^{2} \phi}{\partial Y^{2}}+\left(\frac{N_{t}}{N_{b}}\right) \frac{\partial^{2} \theta}{\partial Y^{2}}\right]-K_{r} \phi^{P}$

Boundary conditions,

$\tau \leq 0, U=0, V=0, \theta=0, \varphi=0$ every where
$\tau>0, U=0, V=0, \theta=0, \varphi=0$ at $X=0$
$U=0, N=-\frac{1}{2} \frac{\partial u}{\partial y}, T=1, C=1 \quad$ at $y=0$
$U=0, N=0, \quad T=0, C=0 \quad$ at $\quad y \rightarrow \infty$

where the obtained physical parameters are given below:

Grashof number, $G_{r}=\frac{g \beta\left(T_{w}-T_{\infty}\right) v}{U_{0}^{3}}$, mass Grashof number. 
$G_{r}=\frac{g \beta^{*}\left(C_{w}-C_{\infty}\right) v}{U_{0}^{3}}$, micro-rotational number, $\Gamma=\frac{\chi}{\rho v}$, magnetic parameter, $M=\frac{\sigma^{\prime} B_{0}^{2} v}{\rho U_{0}^{2}} \quad, \quad$ Darcy number, $D_{a}=\frac{K^{\prime} U_{0}^{2}}{v^{2}}$, Spin Gradient viscosity, $\Lambda=\frac{\gamma}{\rho j v}$ and Vortex viscosity, $\lambda=\frac{\chi v}{\rho j v_{0}^{2}}$, Prandtl number, $P_{r}=\frac{\rho c_{p} v}{\kappa}$, radiation parameter, $R=\frac{\sigma T_{\infty}^{3}}{k_{1} k}$, heat sink parameter, $Q=\frac{Q_{0} v}{U_{0}^{2} \rho c_{p}}$, Forchheimer number, Eckert number, $E_{c}=\frac{U_{0}^{2}}{c_{p}\left(T_{w}-T_{\infty}\right)}$ and Dufour number, $D_{u}=\frac{D_{m} \kappa_{T}}{c_{s} c_{p} v}\left(\frac{C_{w}-C_{\infty}}{T_{w}-T_{\infty}}\right)$, chemical reaction, $K_{r}=\frac{v K_{c}\left(C_{w}-C_{\infty}\right)^{p-1}}{U_{0}^{2}}$. Lewis number, $L_{e}=\frac{v}{D_{m}}$, Brownian parameter, $N_{b}=\frac{\Gamma D_{B}\left(C_{w}-C_{\infty}\right)}{v}$, thermophoresis parameter $N_{t}=\frac{\Gamma D_{T}}{T_{\infty} v}\left(T_{w}-T_{\infty}\right)$.

\section{SHEAR STRESS, NUSSELT NUMBER AND SHERWOOD NUMBER}

The effects of various parameters on the local and average shear stress have been calculated. The following equations represent the local and average shear stress at the plate. Local shear stress $\tau_{L}=\mu\left(\frac{\partial u}{\partial y}\right)_{y=0}$ and average shear stress $\tau_{A}=\mu \int\left(\frac{\partial u}{\partial y}\right)_{y=0} d x$ which is proportional to $\left(\frac{\partial U}{\partial Y}\right)_{Y=0}$ and $\int_{0}^{100}\left(-\frac{\partial U}{\partial Y}\right)_{Y=0} d X$ respectively. From the temperature field, the effects of various parameters on the local and average heat transfer coefficients have been investigated. The following equations represent the local and average heat transfer rate, which is well known Nusselt number. Local Nusselt number, $N_{u L}=\mu\left(-\frac{\partial T}{\partial y}\right)_{y=0}$ and average Nusselt number, $N_{u A}=\mu \int\left(-\frac{\partial T}{\partial y}\right)_{y=0} d x$ which is proportional to $\left(-\frac{\partial \theta}{\partial Y}\right)_{Y=0}$ and $\int_{0}^{100}\left(-\frac{\partial \theta}{\partial Y}\right)_{Y=0} d X$ respectively. From the concentration field, the effects of various parameters on the local and average mass transfer coefficients have been analysed. The following equations represent the local and average mass transfer rate that is well known Sherwood number. Local Sherwood number, $S_{h L}=\mu\left(-\frac{\partial C}{\partial y}\right)_{y=0}$ and Average Sherwood number, $S_{h A}=\mu \int\left(-\frac{\partial C}{\partial y}\right)_{y=0} d x$ which is proportional to $\left(-\frac{\partial \varphi}{\partial Y}\right)_{Y=0}$ and $\int_{0}^{100}\left(-\frac{\partial \varphi}{\partial Y}\right)_{Y=0} d X$ respectively.

\section{NUMERICAL SOLUTION}

To solve the governing coupled non-dimensional partial differential equations with the associated initial and boundary conditions EFDM has been applied. The explicit finite difference method has been used to solve (6) - (9) subject to the initial and boundary conditions. For this reason, the area within the boundary layer is divided by some perpendicular lines of $Y$-axis, where the normal of the medium is $Y$ - axis as shown in Figure A2. It is assumed that the maximum length of the boundary layer $Y_{\max }=20$ as corresponds to $Y \rightarrow \infty$. i.e. $Y$ vary from 0 to 20 and the number of grid spacing in $Y$ directions are $m(=100)$ and $n(=100)$, with the smaller time step $\Delta \tau=0.005$. Using the explicit finite difference approximation, we have,

Continuity Equation

$\frac{U_{i, j}-U_{i-1, j}}{\Delta X}+\frac{V_{i, j}-V_{i, j-1}}{\Delta Y}=0$

Momentum Equation,

$\frac{U_{i, j}^{\prime}-U_{i, j}}{\Delta \tau}+U_{i, j} \frac{U_{i, j}-U_{i-1, j}}{\Delta X}+V_{i, j} \frac{U_{i, j+1}-U_{i, j}}{\Delta Y}$

$=\frac{U_{i, j+1}-2 U_{i, j}+U_{i, j-1}}{(\Delta Y)^{2}}+G_{r} \theta_{i, j}+G_{c} \phi_{i, j}$

$+(1+\Gamma) \frac{U_{i, j+1}-2 U_{i, j}+U_{i, j-1}}{(\Delta Y)^{2}}+\Gamma \frac{N_{i, j+1}-N_{i, j}}{\Delta Y}$

$-\left(M+\frac{1}{D_{a}}\right) U_{i, j}-\frac{F_{s}}{D_{a}}\left(U_{i, j}\right)^{2}$

Angular Momentum Equation,

$\frac{N_{i, j}^{\prime}-N_{i, j}}{\Delta \tau}+U_{i, j} \frac{N_{i, j}-N_{i-1, j}}{\Delta X}+V_{i, j} \frac{N_{i, j+1}-N_{i, j}}{\Delta Y}$
$=\Lambda \frac{N_{i, j+1}-2 N_{i, j}+N_{1, j-1}}{(\Delta Y)^{2}}-\lambda\left(2 N_{i, j}+\frac{U_{i, j+1}-U_{i, j}}{\Delta Y}\right)$

Energy Equation,

$\frac{\theta_{i, j}^{\prime}-\theta_{i, j}}{\Delta \tau}+U_{i, j} \frac{\theta_{i, j}-\theta_{i-1, j}}{\Delta X}+V_{i, j} \frac{\theta_{i, j+1}-\theta_{i, j}}{\Delta Y}=Q \theta_{i, j}$

$+Q_{1} \phi_{i, j}+\frac{1}{P_{r}}\left(1+\frac{16}{3} R\right) \frac{\theta_{i, j+1}-2 \theta_{i, j}+\theta_{i, j-1}}{(\Delta Y)^{2}}$

$+D_{u} \frac{\phi_{i, j+1}-2 \phi_{i, j}+\phi_{i, j-1}}{(\Delta Y)^{2}}+(1+\Gamma) E_{c}\left(\frac{U_{i, j+1}-U_{i, j}}{\Delta Y}\right)^{2}$

$+\left(\frac{N_{i, j+1}-N_{i, j}}{\Delta Y}\right)^{2}+N_{b}\left(\frac{\theta_{i, j+1}-\theta_{i, j}}{\Delta Y} \cdot \frac{\varphi_{i, j+1}-\varphi_{i, j}}{\Delta Y}\right)$

$+N_{t}\left(\frac{\theta_{i, j+1}-\theta_{i, j}}{\Delta Y}\right)^{2}$ 


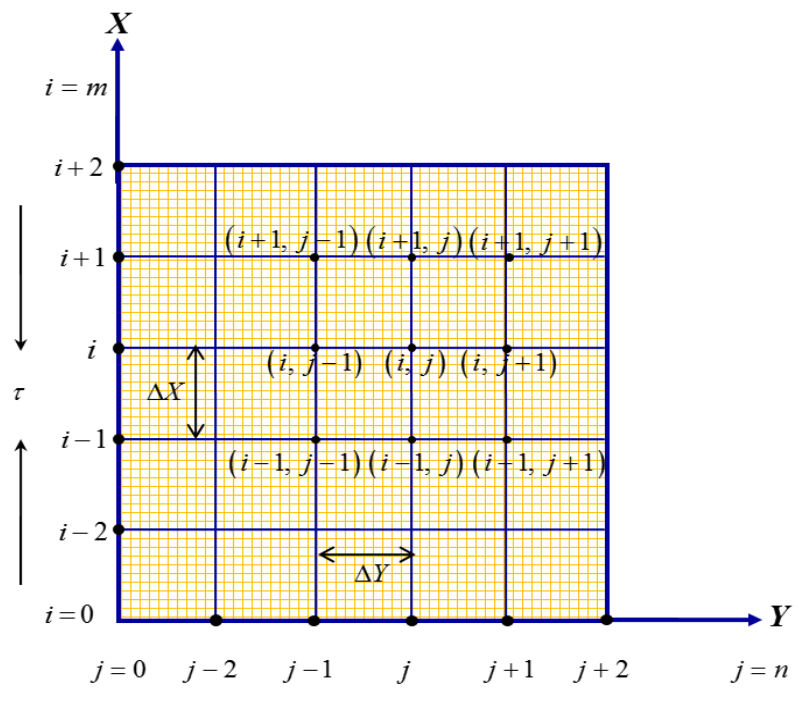

Figure A2. The finite difference space grid

Concentration Equation,

$\frac{\phi_{i, j}^{\prime}-\phi_{i, j}}{\Delta \tau}+U_{i, j} \frac{\phi_{i, j}-\phi_{i-1, j}}{\Delta X}+V_{i, j} \frac{\phi_{i, j+1}-\phi_{i, j}}{\Delta Y}=$

$-K_{r}\left(\phi_{i, j}\right)^{P}+\frac{1}{L_{e}}\left[\frac{\phi_{i, j+1}-2 \phi_{i, j}+\phi_{i, j-1}}{(\Delta Y)^{2}}\right.$

$\left.+\left(\frac{N_{t}}{N_{b}}\right) \frac{\theta_{i, j+1}-2 \theta_{i, j}+\theta_{i, j-1}}{(\Delta Y)^{2}}\right]$

The initial and boundary condition with finite difference scheme as

$$
\begin{aligned}
& U_{i, 0}^{n}=0, N_{i, 0}^{n}=-\frac{1}{2} \frac{U_{i, 0+1}-U_{i, 0}}{(\Delta Y)^{2}}, \theta_{i, 0}^{n}=1, \phi_{i, 0}^{n}=1 \\
& U_{i, L}^{n}=0, N_{i, L}^{n}=0, \theta_{i, L}^{n}=0, \phi_{i, L}^{n}=0 \text { Where, } L \rightarrow \infty
\end{aligned}
$$

where, the subscripts $i$ and $j$ designate the grid points with $X$ and $Y$ coordinates respectively and a value of time, $\tau=n \Delta \tau$, where $n=1,2,3,4 \ldots \ldots$.

\subsection{Stability and convergence test}

The analysis remains incomplete unless the stability and convergence of the finite difference scheme are discussed. For the constant mesh size, the stability criteria of the scheme may be established as follows. The general terms of the Fourier expansion for $U, W, T$ and $C$ at a time arbitrarily called $t=0$ are all $e^{i \alpha X} e^{i \beta Y}$ apart from a constant, where $i=\sqrt{-1}$. A time $t=\tau$, these terms become,

$$
\begin{aligned}
& U: \psi_{1}(\tau) e^{i \alpha X} e^{i \beta Y} \\
& W: \psi_{2}(\tau) e^{i \alpha X} e^{i \beta Y} \\
& \theta: \theta(\tau) e^{i \alpha X} e^{i \beta Y} \\
& C: \varphi(\tau) e^{i \alpha X} e^{i \beta Y}
\end{aligned}
$$

and after a time step, these terms convert to

$$
\left.\begin{array}{l}
U: \psi_{1}^{\prime}(\tau) e^{i \alpha X} e^{i \beta Y} \\
W: \psi_{2}^{\prime}(\tau) e^{i \alpha X} e^{i \beta Y} \\
\theta: \theta^{\prime}(\tau) e^{i \alpha X} e^{i \beta Y} \\
C: \varphi^{\prime}(\tau) e^{i \alpha X} e^{i \beta Y}
\end{array}\right\}
$$

Substituting (17) and (18) to the main equations (13) -(16) we get,

Then

$$
\begin{aligned}
& \frac{\psi_{1}^{\prime}(\tau)-\psi_{1}(\tau)}{\Delta \tau}+U \frac{\psi_{1}(\tau)\left(1-e^{-i \alpha \Delta X}\right)}{\Delta X}+V \frac{\psi_{1}(\tau)\left(e^{i \beta \Delta Y}-1\right)}{\Delta Y} \\
& =G_{r} \theta e^{i \alpha X} e^{i \beta Y}+G_{m} \varphi e^{i \alpha X} e^{i \beta Y}-\left(M+\frac{1}{D_{a}}\right) \psi_{1}(\tau) e^{i \alpha X} e^{i \beta Y} \\
& +(1+\Delta) \frac{\psi_{1}(\tau) e^{i \alpha X} e^{i \beta Y}-2 \psi_{1}(\tau) e^{i \alpha X} e^{i \beta Y}+\psi_{1}(\tau) e^{i \alpha X} e^{i \beta Y}}{(\Delta Y)^{2}} \\
& +\frac{\Delta\left(\psi_{2}(\tau) e^{i \alpha X} e^{i \beta Y}-\psi_{2}(\tau) e^{i \alpha X} e^{i \beta Y}\right)}{\Delta Y}-\frac{F_{s}}{D_{a}}\left(\psi_{1}(\tau) e^{i \alpha X} e^{i \beta Y}\right)^{2} \\
& \Rightarrow \psi_{1}^{\prime}=A_{1} \psi_{1}+A_{2} \psi_{2}+A_{3} \theta+A_{4} \varphi
\end{aligned}
$$

where,

$$
\begin{aligned}
& A_{1}=1+\Delta \tau(1+\Delta) \frac{2(\cos \beta \Delta Y-1)}{(\Delta Y)^{2}}-\left(M+\frac{1}{D_{a}}\right)-\frac{F_{s}}{D_{a}} \\
& -U \frac{\left(1-e^{-i \alpha \Delta X}\right)}{\Delta X}-V \frac{\left(e^{i \beta \Delta Y}-1\right)}{\Delta Y} \\
& A_{2}=\Delta \tau \frac{\Delta\left(e^{i \alpha \Delta Y}-1\right)}{\Delta Y}, A_{3}=\Delta \tau G_{r} \text { and } A_{4}=\Delta \tau G_{m}
\end{aligned}
$$

For the angular velocity,

$$
\begin{aligned}
& \frac{\psi_{2}{ }^{\prime}(\tau)-\psi_{2}(\tau)}{\Delta \tau}+U \frac{\psi_{2}(\tau)\left(1-e^{-i \alpha \Delta X}\right)}{\Delta X}+V \frac{\psi_{2}(\tau)\left(e^{i \beta \Delta Y}-1\right)}{\Delta Y} \\
& =\wedge \frac{2 \psi_{2}(\tau) e^{i \alpha X} e^{i \beta Y}(\cos \beta \Delta Y-1)}{(\Delta Y)^{2}} \\
& -\lambda\left\{2 \psi_{2} e^{i \alpha X} e^{i \beta Y}+\psi_{1} e^{i \alpha X} e^{i \beta Y} \frac{e^{i \beta \Delta Y}-1}{\Delta Y}\right\} \\
& \psi_{2}^{\prime}=A_{5} \psi_{2}-A_{6} \psi_{1}
\end{aligned}
$$

where,

$$
\begin{aligned}
& A_{5}=1+\wedge \frac{2 \Delta \tau(\cos \beta \Delta Y-1)}{(\Delta Y)^{2}} \\
& -U \frac{\left(1-e^{-i \alpha \Delta X}\right)}{\Delta X}-V \frac{\left(e^{i \beta \Delta Y}-1\right)}{\Delta Y}-2 \Delta \tau \lambda \\
& A_{6}=\frac{\Delta \tau \lambda\left(e^{i \alpha \Delta Y}-1\right)}{\Delta Y}
\end{aligned}
$$

For temperature equation, 


$$
\begin{aligned}
& \theta^{\prime}=\theta+\Delta \tau\left[\frac{1}{P_{r}}\left(1+\frac{16 R}{3}\right) \frac{2 \theta(\cos \beta \Delta Y-1)}{(\Delta Y)^{2}}+D_{u} \frac{2 \varphi(\cos \beta \Delta Y-1)}{(\Delta Y)^{2}}\right. \\
& +Q \theta-U \frac{\left(1-e^{-i \alpha \Delta X}\right)}{\Delta X}-V \frac{\left(e^{i \beta \Delta Y}-1\right)}{\Delta Y}+N_{t} T\left(\frac{e^{i \beta \Delta Y}-1}{\Delta Y}\right)^{2} \\
& \left.+N_{b} C\left(\frac{e^{i \beta \Delta Y}-1}{\Delta Y}\right)^{2}\right]+E_{c}\left\{\frac{\psi_{1}\left(e^{i \beta \Delta Y}-1\right)}{\Delta Y}\right\}^{2}+\Delta \tau Q_{1} \varphi \\
& \left.+\Delta \tau D_{u} \varphi \frac{2 \Delta \tau(\cos \beta \Delta Y-1)}{(\Delta Y)^{2}}\right] \\
& \theta^{\prime}=A_{7} \theta+A_{8} \varphi+A_{9} \psi_{1}
\end{aligned}
$$

where,

$$
\begin{aligned}
& A_{7}=1+\frac{\Delta \tau}{P_{r}}\left(1+\frac{16 R}{3}\right) \frac{2(\cos \beta \Delta Y-1)}{(\Delta Y)^{2}}+Q \theta-U \frac{\left(1-e^{-i \alpha \Delta X}\right)}{\Delta X} \\
& -V \frac{\left(e^{i \beta \Delta Y}-1\right)}{\Delta Y} \cdot+N_{t} T\left(\frac{e^{i \beta \Delta Y}-1}{\Delta Y}\right)^{2}+N_{b} C\left(\frac{e^{i \beta \Delta Y}-1}{\Delta Y}\right)^{2} \\
& A_{8}=D_{u} \frac{2 \Delta \tau(\cos \beta \Delta Y-1)}{(\Delta Y)^{2}}+Q_{1} \text { and } A_{9}=E_{c} \Delta \tau \frac{\left(e^{i \beta \Delta Y}-1\right)^{2}}{(\Delta Y)^{2}}
\end{aligned}
$$

For concentration equation,

$$
\begin{aligned}
& \varphi^{\prime}=\varphi+\Delta \tau\left[\frac{1}{L_{e}}\left[\frac{2 \varphi(\cos \beta \Delta Y-1)}{(\Delta Y)^{2}}+\frac{N_{t}}{N_{b}} \frac{2(\cos \beta \Delta Y-1)}{(\Delta Y)^{2}} \theta\right]\right. \\
& \left.-\frac{U\left(1-e^{i \alpha \Delta X}\right)}{\Delta X} \varphi-\frac{V\left(e^{i \beta \Delta Y}-1\right)}{\Delta Y} \varphi\right]-K_{r} \varphi^{P} \\
& \varphi^{\prime}=\varphi\left[1+\frac{2 \Delta \tau}{S_{c}} \frac{(\cos \beta \Delta Y-1)}{(\Delta Y)^{2}}-\Delta \tau K_{r}-\frac{U \Delta \tau\left(1-e^{i \alpha \Delta X}\right)}{\Delta X}\right. \\
& \left.-\frac{V \Delta \tau\left(e^{i \beta \Delta Y}-1\right)}{\Delta Y}\right]+\frac{N_{t}}{N_{b}} \frac{2(\cos \beta \Delta Y-1)}{(\Delta Y)^{2}} \theta \\
& \varphi^{\prime}=A_{10} \varphi+A_{11} \theta
\end{aligned}
$$

where,

$$
\begin{gathered}
A_{10}=1+\frac{2 \Delta \tau}{L_{e}} \frac{(\cos \beta \Delta Y-1)}{(\Delta Y)^{2}}-\Delta \tau K_{r} \\
-\frac{U \Delta \tau\left(1-e^{i \alpha \Delta X}\right)}{\Delta X}-\frac{V \Delta \tau\left(e^{i \beta \Delta Y}-1\right)}{\Delta Y}
\end{gathered}
$$

Equation (18) -(21) can be expressed in the Matrix form,

$$
\left[\begin{array}{c}
\psi_{1}{ }^{\prime} \\
\psi_{2}^{\prime} \\
\theta^{\prime} \\
\varphi^{\prime}
\end{array}\right]=\left[\begin{array}{cccc}
A_{1} & A_{2} & A_{3} & A_{4} \\
-A_{6} & A_{5} & 0 & 0 \\
A_{9} & 0 & A_{7} & A_{8} \\
0 & 0 & A_{11} & A_{10}
\end{array}\right]\left[\begin{array}{c}
\psi_{1} \\
\psi_{2} \\
\theta \\
\varphi
\end{array}\right]
$$

i.e. $\eta^{\prime}=T^{\prime} \eta$

For obtaining the stability condition, Eigenvalues of the amplification matrix $T^{\prime}$ must be finding out. It is a fourth order square matrix. For this explicit finite difference solution, the dimensionless time difference $\Delta \tau$ is very small i.e. tends to zero. Under this condition, $A_{2} \rightarrow 0, A_{3} \rightarrow 0, A_{4} \rightarrow 0, A_{6} \rightarrow 0$, $A_{8} \rightarrow 0, A_{9} \rightarrow 0$ and $A_{11} \rightarrow 0$

$$
\therefore T^{\prime}=\left[\begin{array}{cccc}
A_{1} & 0 & 0 & 0 \\
0 & A_{5} & 0 & 0 \\
0 & 0 & A_{7} & 0 \\
0 & 0 & 0 & A_{10}
\end{array}\right]
$$

After simplification of the matrix, the Eigenvalues are followed, The Eigenvalues of the amplification matrix Tare obtained, $A_{1}=\lambda_{1}, A_{5}=\lambda_{2}, A_{7}=\lambda_{3}$ and $A_{10}=\lambda_{4}$. For stability test, each of the Eigenvalues must not exceed unity in modulus. Under this consideration, the stability conditions are as follows

$$
\left|A_{1}\right| \leq 1,\left|A_{5}\right| \leq 1,\left|A_{7}\right| \leq 1 \text { and }\left|A_{10}\right| \leq 1
$$

Let,

$$
\begin{aligned}
& a_{1}=\Delta \tau, b_{1}=U \frac{\Delta \tau}{\Delta X}, c_{1}=|-V| \frac{\Delta \tau}{\Delta X}, d_{1}=2 \frac{\Delta \tau}{(\Delta Y)^{2}}, \\
& d_{2}=\frac{\Delta \tau}{(\Delta Y)^{3}}, \mathrm{~d}_{3}=\frac{\Delta \tau}{\Delta Y} \text { and } d_{4}=U \frac{\Delta \tau}{\Delta X(\Delta Y)^{2}}
\end{aligned}
$$

$d_{5}=V \frac{2 \Delta \tau}{(\Delta Y)^{3}}$ then, the coefficient of a, b and $\mathrm{c}$ are all nonnegative. So the maximum modulus of $A_{1}, A_{5}, A_{7}$ and $A_{6}$ occurs when $\alpha \Delta Y=n \pi$, where $n$ is an integer and hence $A_{1}$ , $A_{5}, A_{7}$ and $A_{10}$ are real. The values of $\left|A_{1}\right|,\left|A_{4}\right|$ and $\left|A_{6}\right|$ are greater when $n$ is an odd integer, in which case;

$$
\begin{aligned}
& A_{5}=1+\Delta d_{1}+b_{1}+c-2 a \lambda \\
& A_{7}=1-2\left[d_{1} \frac{1}{P_{r}}\left(1+\frac{16}{3} R\right)+\frac{a_{1}}{2} Q+b_{1}+c_{1}\right. \\
& \left.+(1+\Gamma) E c\left(d_{3}\right)^{2}+N_{b} C \frac{d_{1}}{2}+N_{t} T \frac{d_{1}}{2}\right] \\
& A_{10}=1-2\left[d_{1} \frac{1}{L_{e}}+b_{1}+c_{1}+\frac{a_{1}}{2} \mathrm{~K}_{c}\right]
\end{aligned}
$$

Hence the stability conditions of the methods are,

$$
\begin{aligned}
& U \frac{\Delta \tau}{\Delta X}+V \frac{\Delta \tau}{\Delta Y}+\frac{2}{P_{r}}\left(1+\frac{16}{3} R\right) \frac{\Delta \tau}{(\Delta Y)^{2}}-\frac{\Delta \tau Q}{2} \\
& +(1+\Gamma) E c\left(\frac{\Delta \tau}{\Delta Y}\right)^{2}+2 N_{b} \frac{\Delta \tau}{(\Delta Y)^{2}}+2 N_{t} \frac{\Delta \tau}{(\Delta Y)^{2}} \leq 1
\end{aligned}
$$

and $U \frac{\Delta \tau}{\Delta X}+V \frac{\Delta \tau}{\Delta Y}+\frac{2}{L_{e}} \frac{\Delta \tau}{(\Delta Y)^{2}}+\frac{\Delta \tau K_{r}}{2} \leq 1$

When, $\Delta \tau$ and $\Delta Y$ approach to zero then the problem will be converged. With initial boundary conditions and for the values of $\Delta \tau=0.005, \Delta X=0.20$ and $\Delta Y=0.20$ then the problem will be converged at $P_{r} \geq 0.356$ and $L_{e} \geq 0.16$. These converge solutions are shown graphically in Figs.1-24.

\section{RESULTS AND DISCUSSIONS}

The MHD naturally convective heat and mass transfer laminar flow of micropolar fluid in presence of nanofluids 
flow over a vertical porous plate with the effect in presence of magnetic field, thermal radiation, heat source, microrotational, a chemical reaction have been studied numerically. In order to investigate the physical representation of the problem, the numerical values of velocity (U), angular velocity $(\mathrm{N})$, temperature $(\theta)$ and species concentration $(\varphi)$ with the boundary layer have been computed for different parameters. The graphs are illustrated with some fix parameters $\mathrm{Gr}=5.0, \mathrm{Gm}=5.0, \mathrm{Da}=1.0, \mathrm{Fs}=1.0, \mathrm{M}=1.20$, $\wedge=2.00, \lambda=1.50, \operatorname{Pr}=1.38, \mathrm{R}=0.70, \mathrm{EC}=0.00001$, $\mathrm{SC}=16.00, \mathrm{Kr}=0.50, \mathrm{~S}=0.50, \mathrm{Nb}=0.10$ and $\mathrm{Nt}=0.10$. Figure 1 is sketched for the effect of Darcy number (Da). From this figure, it can be noticed that the velocity increase with increasing Da. This is because velocity profile near the wall flattens. The influence of similar parameter on angular velocity is illustrated in Figure 2. At first the angular velocity increase with the increment of $\mathrm{Da}(1.0 \leq M \geq 4.0)$ than at an increase of $\mathrm{Da}$ influenced of angular velocity diminish at $\mathrm{X}=0.1$. Then it has been showing an increasing pattern.

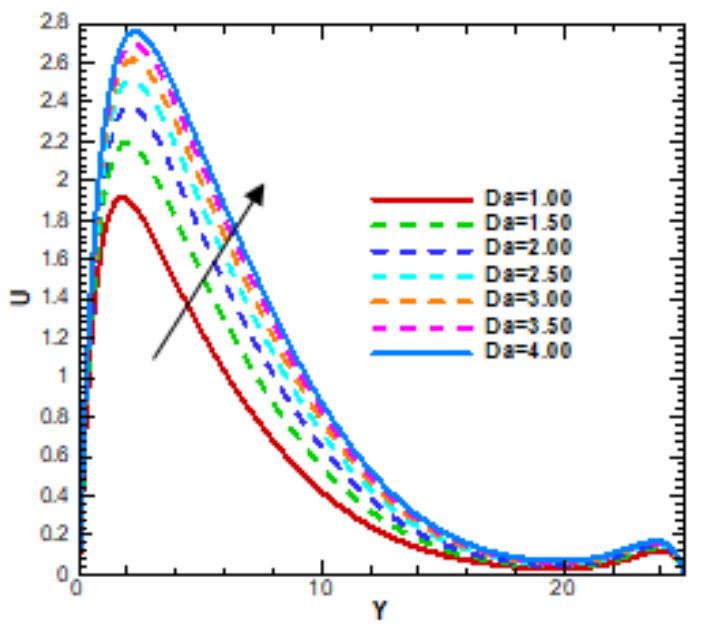

Figure 1. Impact of Da on velocity

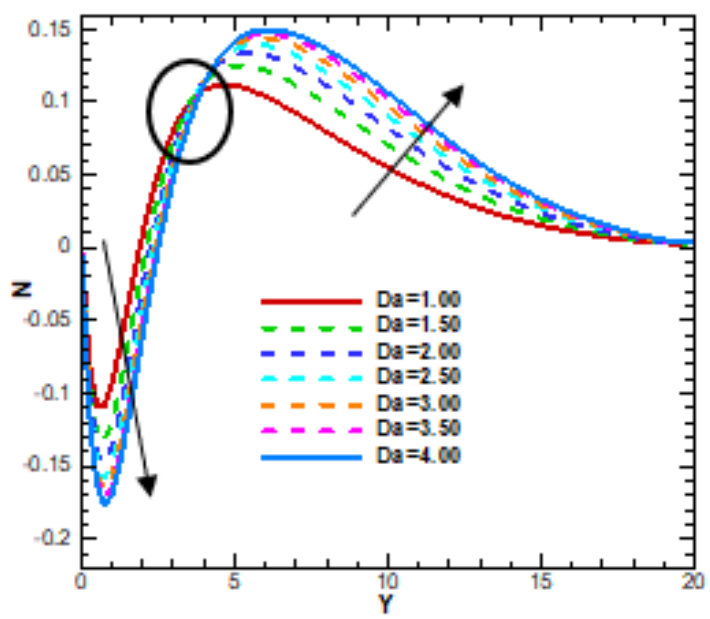

Figure 2. Impact of Da on the angular velocity

Figure 3 and Figure 4 describe that, the increase of magnetic parameter (M) ( $1.20 \leq M \geq 10.50)$ with respect to decreasing pattern of velocity and angular velocity except initially in case of angular velocity. The fact behind this, the presence of a magnetic field in an electrically conducting fluid influenced by Lorentz force, which retracts the flow.

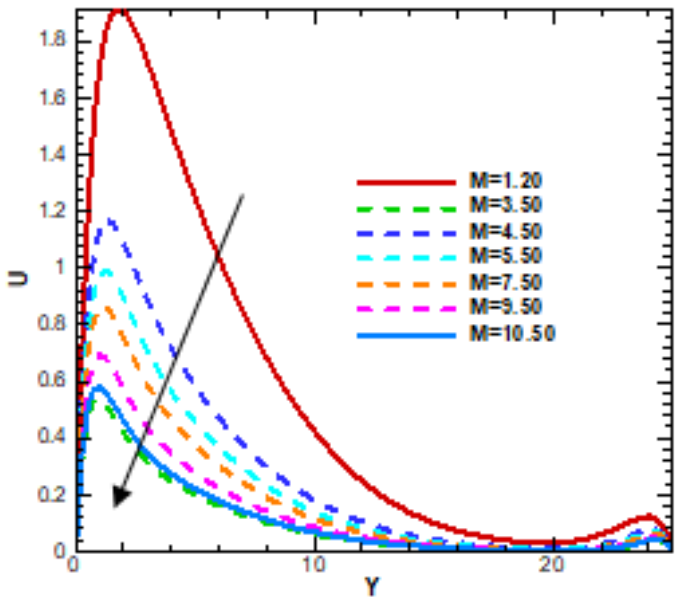

Figure 3. Impact of $\mathrm{M}$ on velocity

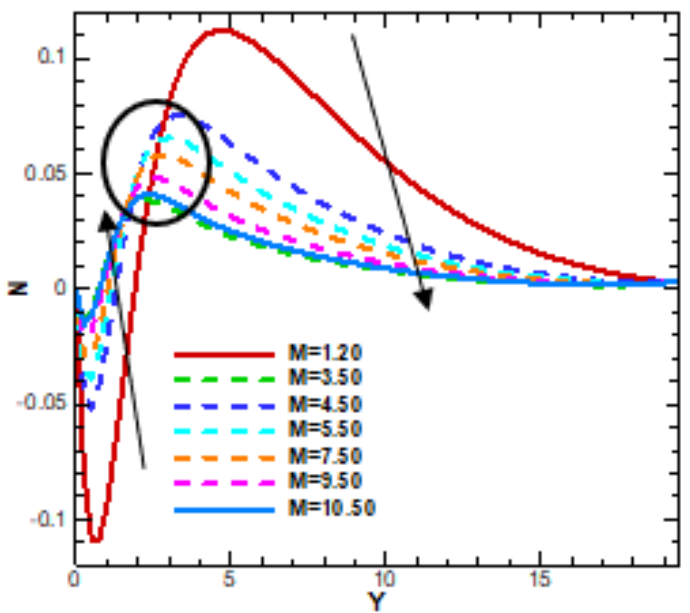

Figure 4. Impact of $\mathrm{M}$ on the angular velocity

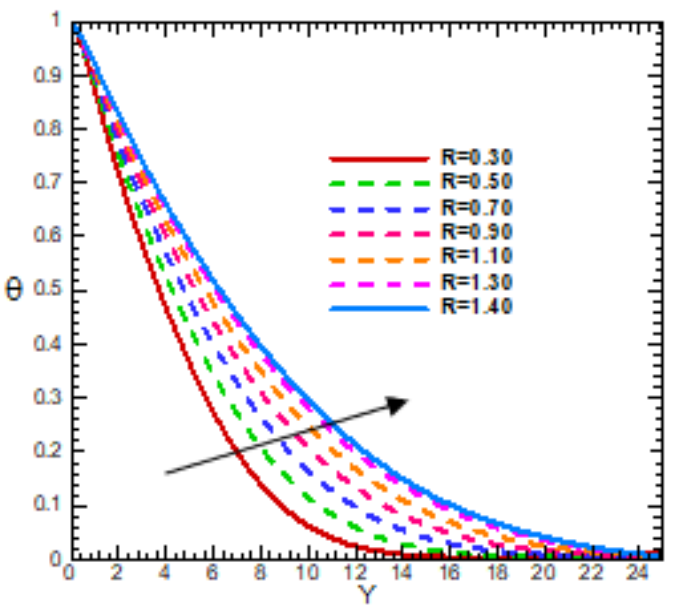

Figure 5. Impact of R on temperature

The effect of radiation parameter $(\mathrm{R})$ on temperature is illustrated in Figure 5 It is seen that temperature profile increase due to rising of $\mathrm{R}(0.50 \leq R \geq 1.40)$. Increasing estimation of $R$ provides more heat to the fluid which raises the temperature and thermal boundary layer. Figure 6 and Figure 7 are plotted for the impact of velocity and angular velocity on $\mathrm{R}$. The opposite behaviours have been shown as increase and decrement of velocity and angular velocity due to elevate of $\mathrm{R}$ are found in here. Figure 8 depicts the temperature 
profiles for different values $(0.10 \leq N b \geq 1.30)$ of Brownian motion $(\mathrm{Nb})$. It is observed that temperature increase with increasing $\mathrm{Nb}$, which indicates enhances the nanoparticles concentration. Through Figure 9 and Figure 10, we elucidated the domination of thermophoresis parameter $(\mathrm{Nt})$ on concentration and angular velocity. Here we detect a rise in the $\mathrm{Nt}(0.10 \leq N t \geq 1.30)$ with enhance of concentration and angular velocity. In Figure 10, near the plate, the angular velocity diminishes with the soar of $\mathrm{Nt}$ and after few time near at $\mathrm{Y}=2.0, \mathrm{X}=0.0$ the profiles increase.

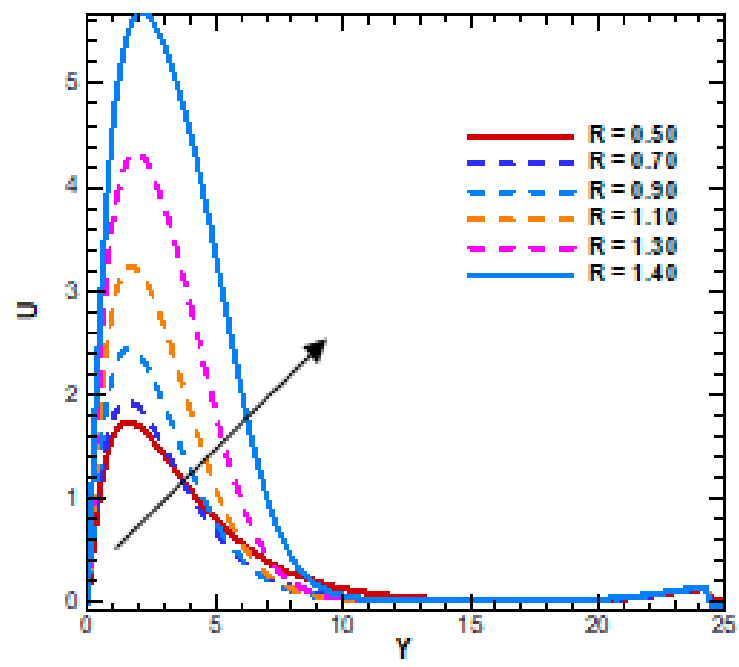

Figure 6. Impact of R on velocity

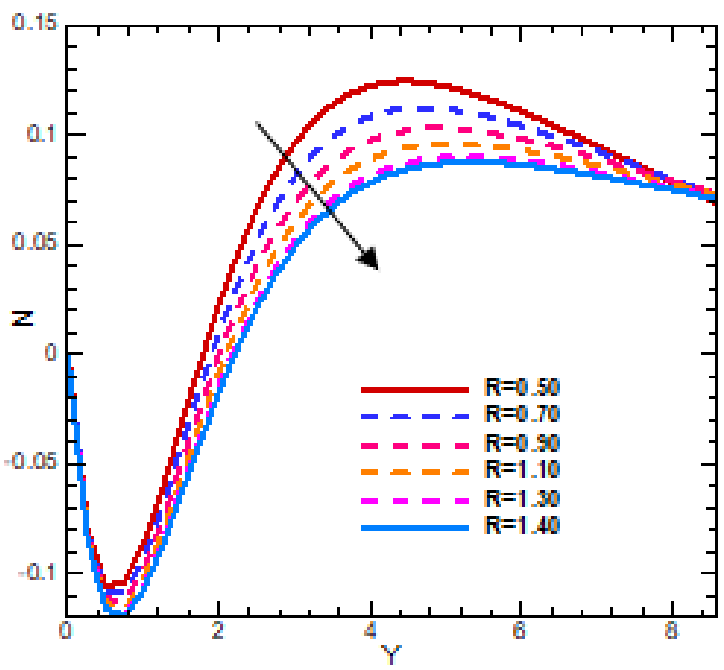

Figure 7. Impact of R on the angular velocity

The graph of Lewis number (Le) is depicted in Figure 11. We eye that an enrichment of Le $(10 \leq L e \geq 17)$ depreciates the concentration distribution. The reason behind this Le expresses the relative contribution of thermal diffusion to mass diffusion in the boundary region. Impact of increasing value of Le will reduce thermal boundary layer thickness as well as concentration. Figure 12 elucidates the nature of concentration for ascending values of destructive chemical reaction $(\mathrm{Kr})$. It is seen that decline of concentration with $\mathrm{Kr}$. Physically, chemical reaction parameter expresses consumption of chemical therefore results shows the decreasing the concentration.

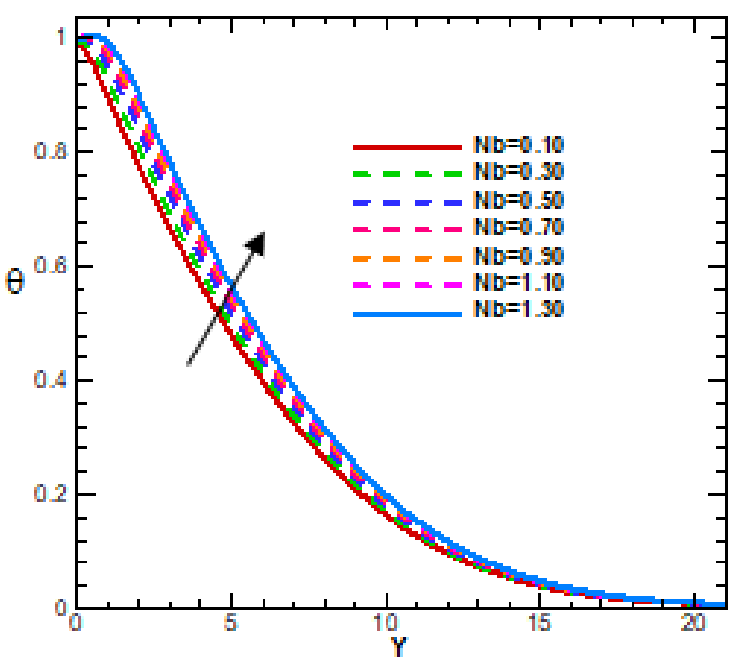

Figure 8. Impact of $\mathrm{Nb}$ on temperature

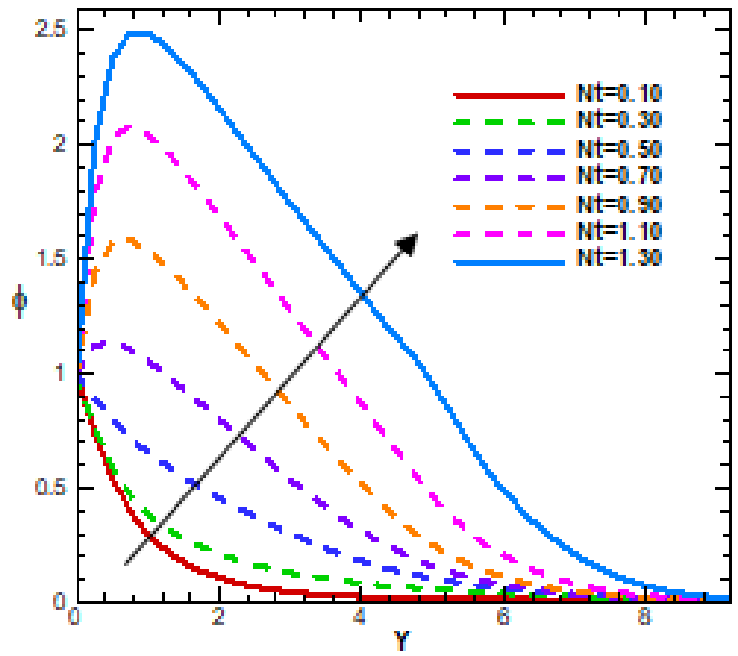

Figure 9. Impact of $\mathrm{Nt}$ on the concentration

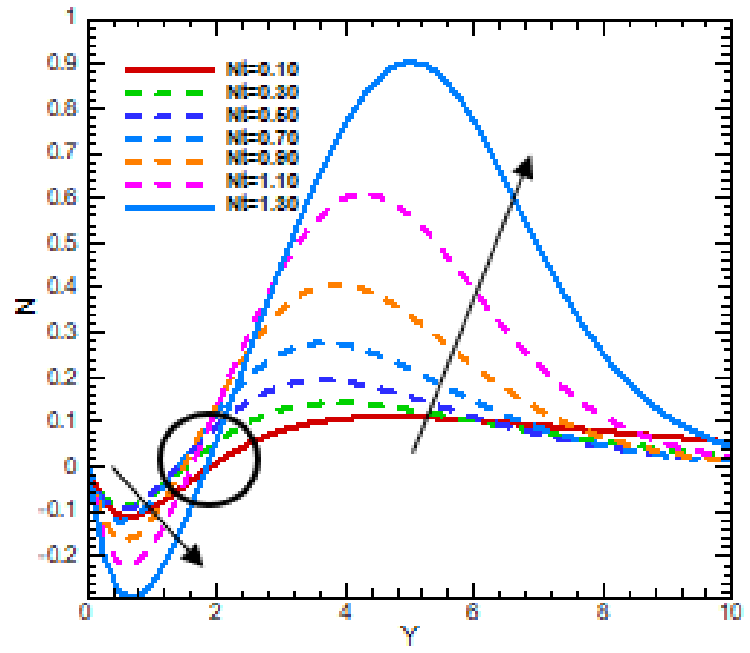

Figure 10. Impact of $\mathrm{Nt}$ on the angular velocity

The angular velocity versus vortex viscosity is demonstrated through Figure 13 Near the plate for an increase of vortex viscosity $(1.50 \leq \lambda \geq 7.0)$, the angular profile decreases and after a time at $\mathrm{Y}=2.0$ and $\mathrm{X}=0.00$ the profiles increase. Here, the angular velocity distribution decreasing for 
increasing value of vortex viscosity.

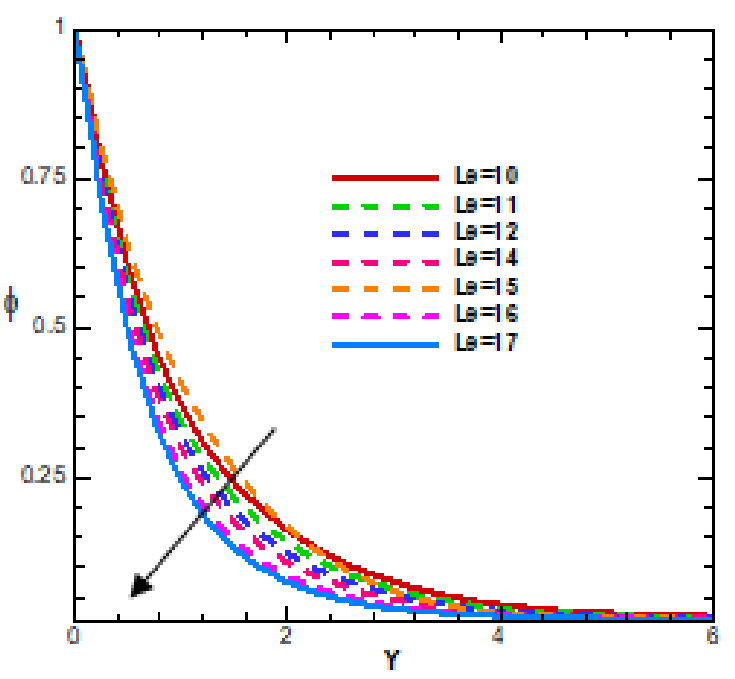

Figure 11. Impact of $\mathrm{Nt}$ on the concentration

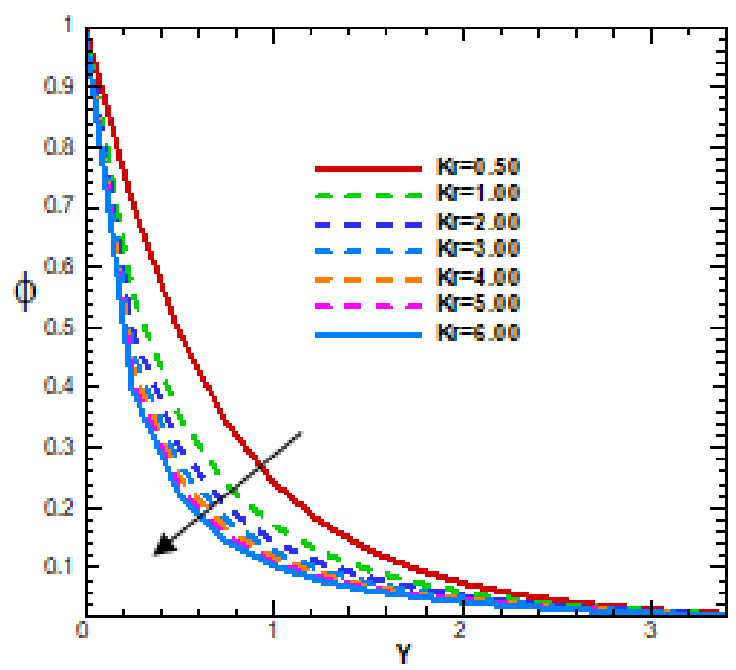

Figure 12. Impact of $\mathrm{Kr}$ on the concentration

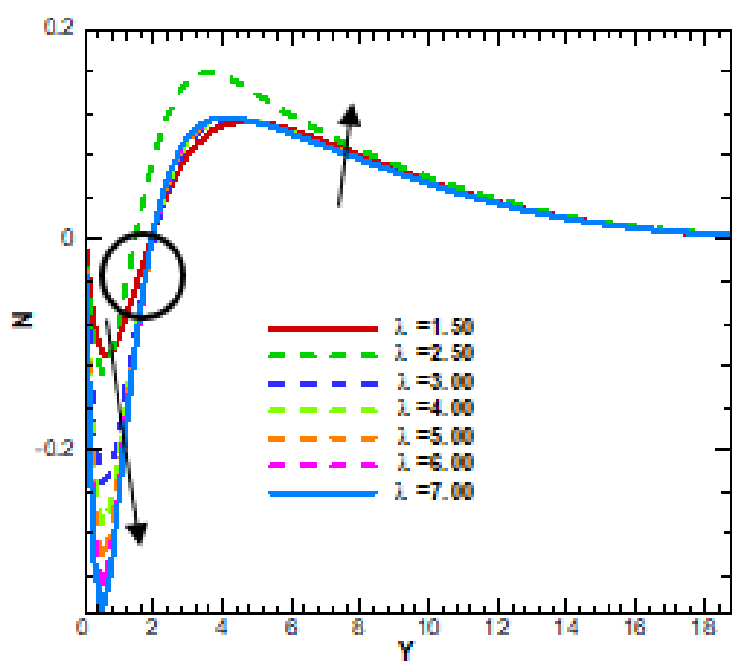

Figure 13. Impact of $\lambda$ on angular velocity

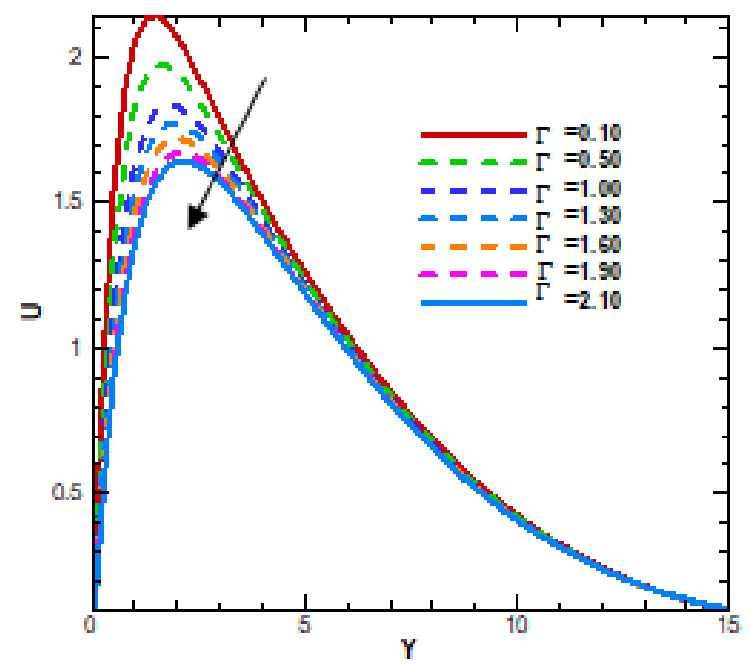

Figure 14. Impact of $\Gamma$ on velocity

Further, Figure 14 and Figure 15 respectively show the ascending profile of micro-rotational number $(\Gamma)$. It is seen that $\Gamma$ ascents, velocity and angular velocity descent.

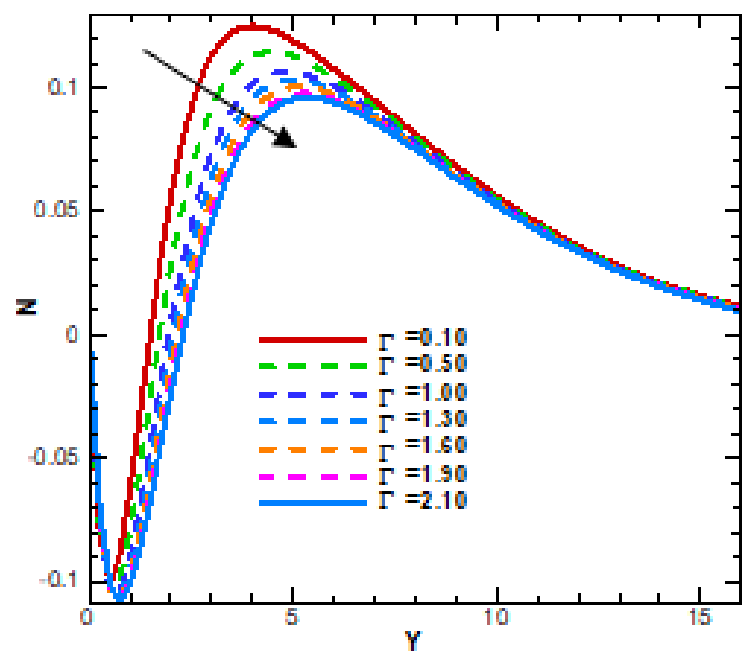

Figure 15. Impact of $\Gamma$ on angular velocity

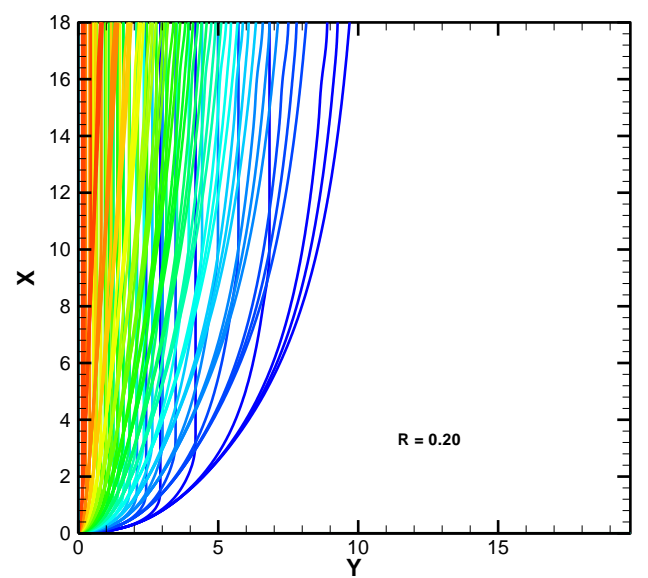

Figure 16. Isotherm lines for $\mathrm{R}=0.20$ with $\tau=1-10$ 


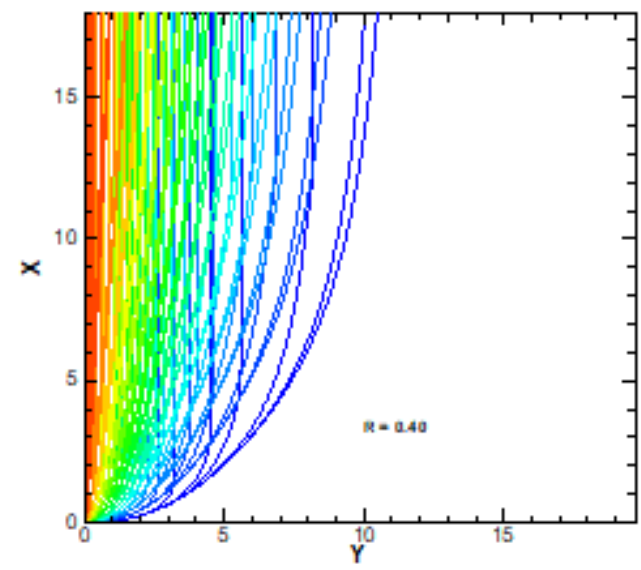

Figure 17. Isotherm lines for $\mathrm{R}=0.40$ with $\tau=1-10$

Figure 16 and Figure 17 illustrate the thermal boundary thickness for the difference values of $\mathrm{R}(0.20$ and 0.40$)$. For the increase of thermal radiation (R), the thermal boundary layer thicknesses of fluid have been expanded for an increase of temperature. Figure 18 and Figure 19 illustrate the momentum boundary thickness for the difference values of $\mathrm{R}$ $(0.20$ and 0.40$)$. For the increase of thermal radiation $(R)$, the momentum boundary layer thicknesses of fluid have been expanded for an increase of temperature.

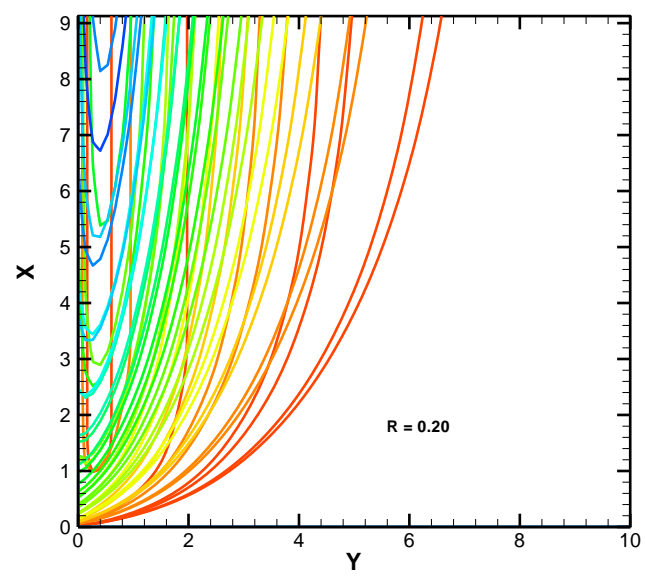

Figure 18. Stem lines for $\mathrm{R}=0.20$ with $\tau=1-10$

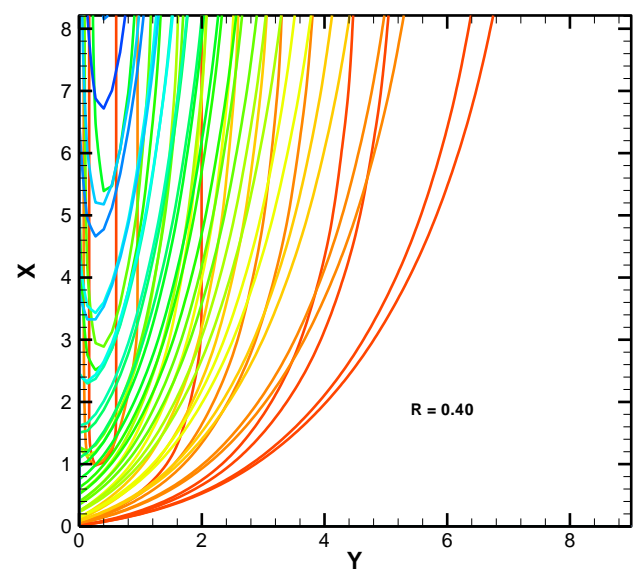

Figure 19. Isotherm lines for $\mathrm{R}=0.40$ with $\tau=1-10$

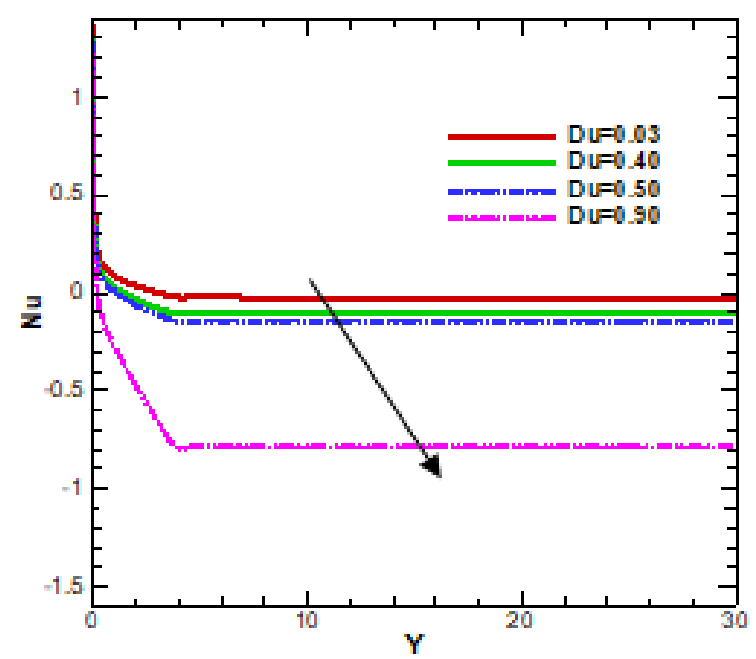

Figure 20. Impact of Du on Nusselt number

From Figure 20, it is noticed an increase in the values of Dufour number created in the reduction of the Nusselt number. The influence of Dufour number $\left(D_{u}\right)$ on Sherwood number $\left(\mathrm{S}_{\mathrm{h}}\right)$ is given in Figure 21. It is evident from this figure $\mathrm{Du}$ enhances by the increasing Sherwood number. Figure 22 Indicates the impact Lewis number on Sherwood number profiles with a rising pattern of $S_{h}$ with respect to Lewis number. Skin friction increased with increasing of Darcy number is shown in Figure. 23 Magnetic parameter is addressed opposite pattern with respect to skin friction in Figure 24.

Table 1. Computations are showing the increase of Nusselt number $\left(N_{u}\right)$ for the increase of $N_{t}$ for $P_{r}=0.71, L_{e}=10.00$ and $\tau=10.00$

\begin{tabular}{cccccc}
\hline$N_{t}$ & $N_{u}$ & $N_{u}$ & $N_{u}$ & $N_{u}$ & $N_{u}$ \\
& $N_{b}=0.10$ & $N_{b}=0.20$ & $N_{b}=0.30$ & $N_{b}=0.40$ & $N_{b}=0.50$ \\
\hline 0.10 & 0.05976 & 0.00297 & -0.01678 & -0.02674 & -0.02267 \\
0.20 & 0.13674 & 0.04906 & -0.01147 & -0.03257 & -0.02035 \\
0.30 & 0.20364 & 0.11765 & 0.03034 & -0.01897 & -0.01687 \\
0.40 & 0.27813 & 0.11862 & 0.09882 & 0.02213 & -0.01364 \\
0.50 & 0.31364 & 0.25804 & 0.16998 & 0.08893 & 0.08034 \\
\hline
\end{tabular}

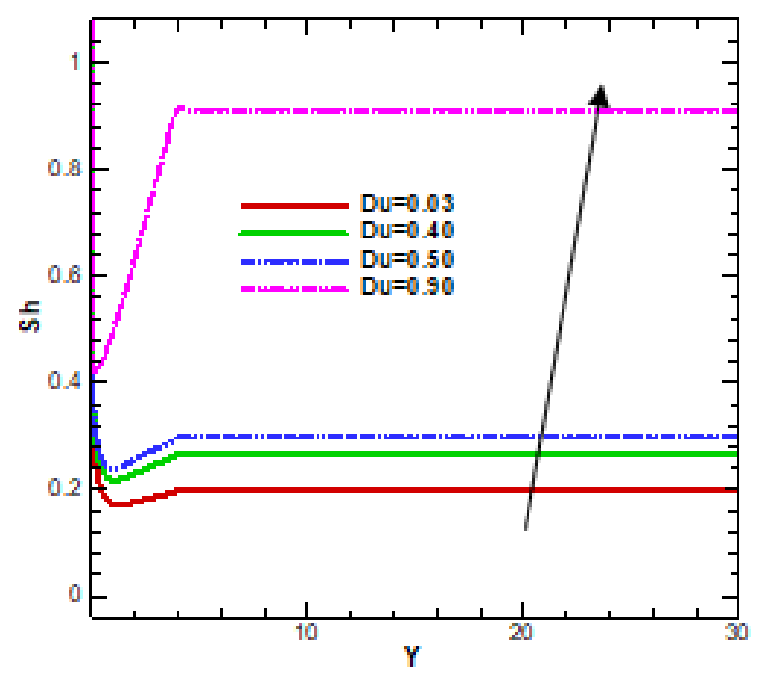

Figure 21. Impact of Du on Sherwood number 


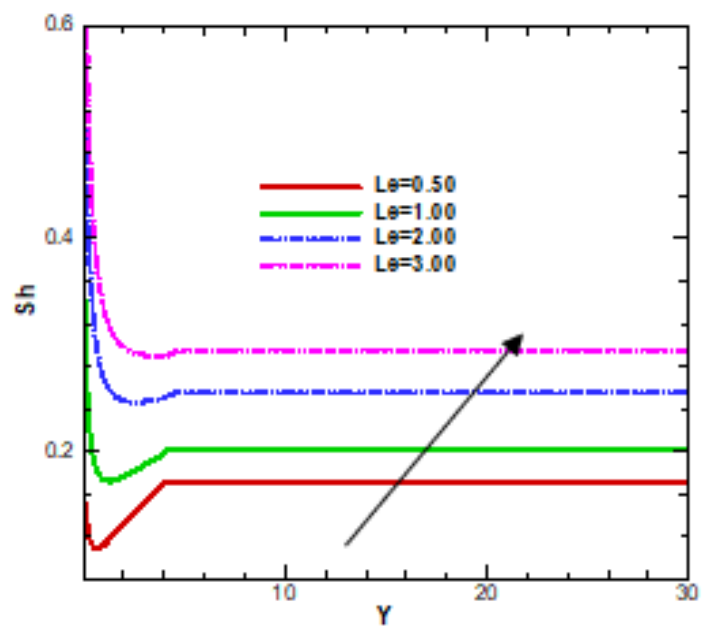

Figure 22. Impact of Le on Sherwood number

Table 2. Computations are showing the decreased of Sherwood number $\left(S_{h}\right)$ for the increase of $N_{t}$ for

$$
P_{r}=0.71, L_{e}=10.00 \text { and } \tau=10.00
$$

\begin{tabular}{cccccc}
\hline$N_{t}$ & $S_{h}$ & $S_{h}$ & $S_{h}$ & $S_{h}$ & $S_{h}$ \\
& $N_{b}=0.1$ & $N_{b}=0.2$ & $N_{b}=0.3$ & $N_{b}=0.4$ & $N_{b}=0.5$ \\
\hline 0.10 & 0.20348 & 0.22648 & 0.25974 & 0.26998 & 0.30145 \\
0.20 & 0.07954 & 0.18946 & 0.20365 & 0.21445 & 0.26587 \\
0.30 & -0.13578 & 0.14668 & 0.19756 & 0.2103 & 0.23587 \\
0.40 & -0.40578 & -0.02354 & 0.11645 & 0.17235 & 0.21242 \\
0.50 & -0.75893 & -0.15879 & 0.05445 & 0.12445 & 0.19864 \\
\hline
\end{tabular}

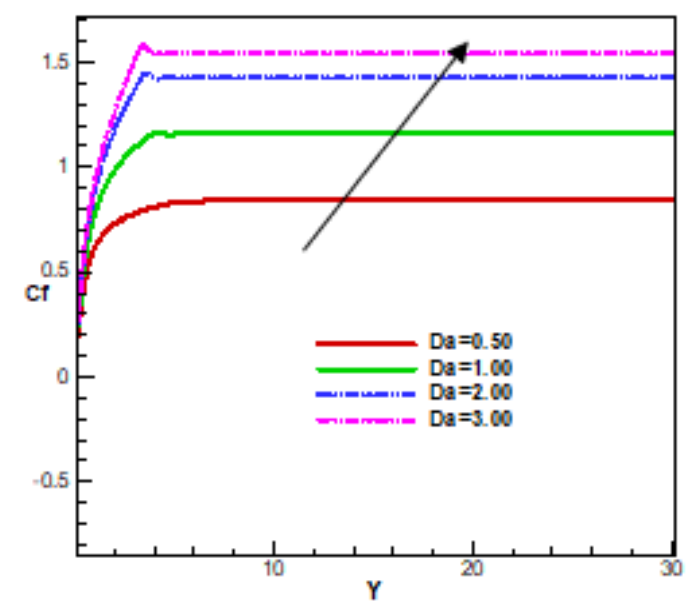

Figure 23. Impact of Da on Skin Friction

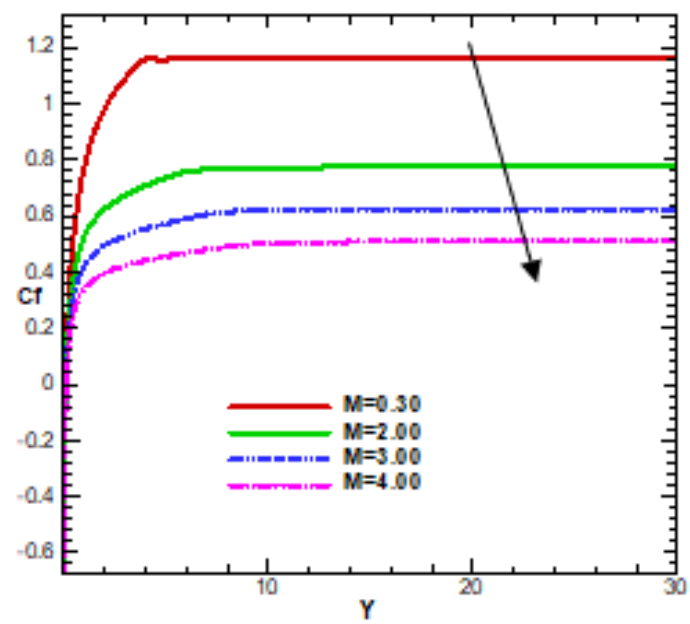

Figure 24. Impact of $M$ on Skin Friction

\section{CONCLUSIONS}

The numerical solution of micropolar fluid with nanoparticle towards a moving semi-infinite vertical porous plate with thermal radiation, heat source, MHD is analysed. The results are presented graphically with various system parameters. Form the graphical representation, the following result deduced from our study:

- The behaviour of velocity distribution decrease for the raising value of magnetic parameter, radiation parameter, micro-rotational number and increase for Darcy number.

- The concentration parameter enhances for the improving values of thermophoresis parameter and it shows decreasing behaviour for Lewis number and chemical reaction.

- Angular velocity profile ascents for escalating Darcy number and thermophoresis parameter while it diminishes for enhancing magnetic parameter, radiation parameter and micro-rotational number.

- Nusselt number decrease for increasing Dufour number.

- Sherwood number is an increasing function of Dufour number and Lewis number.

- The Skin friction distribution increases for aiding Darcy number and decrease for opposing value of the magnetic parameter

\section{REFERENCES}

[1] Eringen AC. (1966). Theory of micropolar fluids. Journal of Mathematics and Mechanics 16: 1-18. https://doi.org/10.1512/iumj.1967.16.16001

[2] Hudimoto B, Tokuoka T. (1969). Two-dimensional shear flows of linear micropolar fluids. Int. J. Eng. Sci. 7: 515522. https://doi.org/10.1016/0020-7225(69)90036-6

[3] Lockwood F, Benchaita F, Friberg S. (1987). Study of lyotropic liquid crystal in viscometric flow and elasto hydrodynamics contact. Tribol. Trans. 30: 539-548. https://doi.org/10.1080/05698198708981790

[4] Ariman T, Turk MA, Sylvester ND. (1974). On steady and pulsatile flow of blood. J. Appl. Mech. 41: 1-7. https://doi.org/10.1115/1.3423224

[5] Eringen AC. (1976) Polar and nonlocal field theories. Academic Press, New York, p. 288.

[6] Ariman T, Turk MA, Sylvester ND. (1974), Microcontinuum fluid mechanics - a review. Int. J. Eng. Sci. 12: 273-293. https://doi.org/10.1016/00207225(73)90038-4

[7] Crane LJ. (1970). Flow past a stretching plate. Z. Angew. Math. Phys. 21: 645-647. http://dx.doi.org/10.1007/BF01587695

[8] Kelson NA, Desseaux A. (2001). Effect of surface condition on flow of micropolar fluid driven by a porous stretching sheet. Int. J. Eng. Sci. 39: 1881-1897. https://doi.org/10.1016/S0020-7225(01)00026-X

[9] Mohammadein AA, Gorla RSR. (2001). Heat transfer in a micropolar fluid over a stretching sheet with viscous dissipation and internal heat generation. Int. J. Num. Methods Heat Fluid Flow 11: 50-58. https://doi.org/10.1108/09615530110364088

[10] Hussain ST, Nadeem S, Rizwan ULH. (2014). Modelbased analysis of micropolar nanofluid flow over a 
stretching surface. Eur. Phys. J. Plus 129(8): 1-10. https://doi.org/10.1140/epjp/i2014-14161-8

[11] Nazar R, Amin N, Filip D, Pop I. (2004). Stagnation point flow of a micropolar fluid towards a stretching sheet. Int. J. Non-Linear Mech. 39(7): 1227-35. https://doi.org/10.1016/j.ijnonlinmec.2003.08.007

[12] Nazar R, Ishak A, Pop I. (2008). Unsteady boundary layer flow over a stretching sheet in a micropolar fluid. Int. J. Math. Phys. Eng. Sci. 2(3): 161-5.

[13] Bhargava R, Kumar L, Takhar HS. (2003). Finite element solution of mixed convection micropolar fluid driven by a porous stretching sheet. Int. J. Eng. Sci. 41: 2161-2178. 7225(03)00209-X

[14] Bhargava R, Sharma S, Takhar HS, Beg OA, Bhargava P. (2007). Numerical solutions for microplar transport phenomena over a nonlinear stretching sheet. Nonlinear Anal., Model. Control 12: 45-63.

[15] Erickson LE, Fan LT, Fox VG. (1966). Heat and mass transfer on moving continuous flat plate with suction or injection. Ind. Eng. Chem. Fundam. 5: 19-25. https://doi.org/10.1021/i160017a004

[16] Fox VG, Erickson LE, Fan LT. (1968). Methods for solving the boundary layer equations for moving continuous flat surfaces with suction and injection. AIChE J. 14: 726-736. https://doi.org/10.1002/aic.690140510

[17] Dutta BK, Roy P, Gupta AS. (1985). Temperature field in the flow over a stretching sheet with uniform heat flux. Int. Commun. Heat Mass Transf. 12: 89-94. https://doi.org/10.1016/0735-1933(85)90010-7

[18] Hayat T, Javed T, Abbas Z. (2009). MHD flow of a micropolar fluid near a stagnation point towards a nonlinear stretching surface. Nonlinear Anal., Real World Appl.

$10(3): 1514-1526$ https://doi.org/10.1016/j.nonrwa.2008.01.019

[19] Ishak A. (2010). Thermal boundary layer flow over a stretching sheet in a micropolar fluid with radiation effect. Meccanica 45: 367-373 https://doi.org/10.1007/s11012-009-9257-4

[20] Latiff NAA, Uddin MJ, Bég OA, Ismail AI. (2015). Unsteady forced bioconvection slip flow of a micropolar nanofluid from a stretching/shrinking sheet. Proceedings of the Institution of Mechanical Engineers Part N Journal of Nanoengineering 230(4): 177-187. https://doi.org/10.1177/1740349915613817

[21] Abel MS, Siddheshwar PG, Mahesha N. (2011). Numerical solution of the momentum and heat transfer equations for a hydromagnetic flow due to a stretching sheet of a non-uniform property micropolar liquid. Appl. Math. Comput. 217: 5895-5909. https://doi.org/10.1016/j.amc.2010.12.081

[22] Abbas Z, Naveed M, Sajid M. (2013). Heat transfer analysis for stretching flow over a curved surface with magnetic field. Journal of Engineering Thermophysics 22(4): 37-345. https://doi.org/ $10.1134 / \mathrm{S} 1810232813040061$

[23] Naveed M, Abbas Z, Sajid M. (2016). Hydromagnetic flow over an unsteady curved stretching surface. Eng. Sci. Technol. Int. J. 19(2): 841-5. https://doi.org/10.1016/j.jestch.2015.11.009

[24] Naveed M, Abbas Z, Sajid M. (2016). MHD flow of a micropolar fluid due to a curved stretching sheet with thermal radiation. J Appl Fluid Mech. 9(1): 131-138. https://doi.org/10.18869/acadpub.jafm.68.224.23967

[25] Bilal M, Hussain S, Sagheer M. (2017). Boundary layer flow of magneto-micropolar nanofluid flow with Hall and ion-slip effects using variable thermal diffusivity. Bulletin of the Polish Academy of Sciences Technical Sciences 65(3): 383-390. https://doi.org/10.1515/bpasts2017-0043

[26] Arifuzzaman SM, Rana BMJ, Ahmed R, Ahmmed SF. (2017). Cross diffusion and MHD effect on a high order chemically reactive micropolar fluid of naturally convective heat and mass transfer past through an infinite vertical porous medium with a constant heat sink. AIP Conference Proceedings, 1851, 020006. https://doi.org/10.1063/1.4984635

[27] Arifuzzaman SM, Khan MS, Hossain KE, Islam MS, Akter S, Roy R. (2017). Chemically reactive viscoelastic fluid flow in presence of nano particle through porous stretching sheet. Frontiers in Heat and Mass Transfer 9(5): 1-11. http://dx.doi.org/10.5098/hmt.9.5

[28] Khan MS, Rahman MM, Arifuzzaman SM, Biswas P, Karim I. (2017), Williamson fluid flow behaviour of MHD convective and radiative Cattaneo-Christov heat flux type over a linearly stretched surface with heat generation, viscous dissipation and thermal-diffusion. Frontiers in Heat and Mass Transfer 9(15): 1-11. http://dx.doi.org/10.5098/hmt.9.15

[29] Biswas P, Arifuzzaman SM, Karim I, Khan MS. (2017). Impacts of magnetic field and radiation absorption on mixed convective Jeffrey Nano fluid flow over a vertical stretching sheet with stability and convergence analysis. Journal of nanofluids 6(6): 1082-1095. https://doi.org/10.1166/jon.2017.1407

[30] Arifuzzaman SM, Khan MS, Islam MS, Islam MM, Rana BMJ, Biswas P, Ahmmed SF. (2017). MHD Maxwell fluid flow in presence of nano-particle through a vertical porous-plate with heat- generation, radiation absorption and chemical reaction. Frontiers in Heat and Mass Transfer 9 (25): 1-14. http://dx.doi.org/10.5098/hmt.9.25

[31] Arifuzzaman SM, Khan MS, Mehedi MFU, Rana BMJ, Ahmmed SF. (2018). Chemically reactive and naturally convective high-speed MHD fluid flow through an oscillatory vertical porous-plate with heat and radiation absorption effect. Engineering Science and Technology, an International Journal 21(2): 215-228. https://doi.org/10.1016/j.jestch.2018.03.004

[32] Vedavathi N, Ramakrishna K, Reddy KJ. (2015). Radiation and mass transfer effects on unsteady MHD convective flow past an infinite vertical plate with Dufour and Soret effects. Ain Shams Engineering Journal 6: 363-3. https://doi.org/10.1016/j.asej.2014.09.009

[33] Wernik J, Grabowski M, Wołosz KJ. (2018). Thermal analysis of radiator under natural and forced convection conditions using numerical simulation and thermography. Chemical Engineering Transactions 70: 1501-1506. https://doi.org/10.3303/CET1870251

[34] Oravec J, Bakošová M, Vasičkaninová A, Mészáros A. (2018). Robust model predictive control of a plate heat exchanger. Chemical Engineering Transactions 70: 2530. https://doi.org/10.3303/CET1870005 


\section{NOMENCLATURE}

$B$ 。

$C_{f}$

$C_{p}$

$D_{a}$

$D_{B}$

$D_{u}$

$E_{c}$

$G_{r}$

$G_{c}$

j

$K^{\prime}$

$K_{r}$

$k_{e}$

$L_{e}$

$N_{b}$

$N_{t}$

$\frac{N_{u}}{N}$

$P_{r}$

$q_{r}$

$Q_{1}{ }^{*}$ magnetic component, $\left(\mathrm{Wb} \mathrm{m}^{-2}\right)$

skin-friction, (-)

specific heat at constant pressure, $\left(\mathrm{J} \mathrm{kg}^{-1} \mathrm{~K}^{-1}\right)$

Darcy number, (-)

The Brownian diffusion coefficient, (-)

Dufour number, (-)

Eckert number, (-)

Grashof number, (-)

modified Grashof number, (-)

Micro-inertia density

the permeability of the porous medium, (-)

chemical reaction parameter, (-)

mean absorption coefficient

Lewis number, (-)

The Brownian parameter, (-)

thermophoresis parameter, (-)

local Nusselt number, (-)

Angular velocity

Prandtl number, (-)

unidirectional radiative heat flux, $\left(\mathrm{kg} \mathrm{m}^{-2}\right)$

radiation absorption, (-) heat absorption quantity, (-)

Sherwood number, (-)

Fluid temperature, $(\mathrm{K})$

The temperature at the plate surface, $(\mathrm{K})$

ambient temperature as y tends to infinity,

(K)

uniform velocity

velocity components

Cartesian coordinates

\section{Greek symbols}

$\beta \quad$ thermal expansion coefficient

$\beta^{*} \quad$ concentration expansion co-efficient

$\gamma \quad$ spin gradient viscosity

$\kappa \quad$ thermal conductivity, $\left(\mathrm{Wm}^{-1} \mathrm{~K}^{-1}\right)$

$\mu \quad$ dynamic viscosities

$v \quad$ kinematic viscosity, $\left(\mathrm{m}^{2} \mathrm{~s}^{-1}\right)$

$\rho \quad$ the density of the fluid, $\left(\mathrm{kg} \mathrm{m}^{-3}\right)$

$\sigma_{s} \quad$ Stefan-Boltzmann constant, $5.6697 \times$ $10^{-8}\left(\mathrm{~W} / \mathrm{m}^{2} \mathrm{~K}^{4}\right)$

electric conductivity

vortex viscosity 